\title{
Ecosistema emprendedor y segmentación de emprendedores del sector hotelero
}

\section{Entrepreneurial ecosystem and segmentation of entrepreneurs in the hotel sector}

\author{
Mónica Jackeline Leoro Llerena ${ }^{1}$ \\ mleorol@ulvr.edu.ec \\ Olmedo Farfán González ${ }^{2}$ \\ olmedo.farfan@uteg.edu.ec
}

\section{RESUMEN}

Recibido: 1/07/2017, Aceptado: 1/09/2017

Conociendo que el emprendimiento es un factor clave en el desarrollo económico de los países y considerando que en Ecuador la mayoría de los emprendimientos que existen se han generado por necesidad y muchos carecen de un valor agregado que los distinga y asegure su permanencia en el tiempo; esta investigación tiene como objetivo determinar los factores que condicionan el ecosistema emprendedor y la segmentación de emprendedores en el sector hotelero de Guayaquil, fundamentado en el marco teórico de la teoría institucional. El estudio es un diseño no-experimental transeccional correlacional, donde se desea determinar cuáles de los factores institucionales condicionan ese ecosistema y sobre esa perspectiva distinguir la estratificación de los emprendedores. Para el análisis se emplean métodos empíricos de investigación como el diseño transeccional correlacional, la encuesta, indicadores de percepción ponderados y el método de análisis de componentes principales.

Palabras clave: ecosistema, emprendedor, segmentación, no-experimental, transeccional, correlacional

\begin{abstract}
Knowing that entrepreneurship is a key factor in economic development of the countries and considering that in Ecuador most of the enterprises that exist have been generated out of necessity and many lack a value added that distinguishes them and ensures their permanence over time; is research aims to determine the factors that condition the Entrepreneurial ecosystem and the segmentation of entrepreneurs in the sector hotel of Guayaquil, based on the theoretical framework of institutional theory. The study is a non-experimental correlational cross design, where wants to determine which of the institutional factors condition that ecosystem and, on that perspective, distinguish the stratification of entrepreneurs. Empirical research methods are used for the analysis as the correlational cross design, the survey, indicators of Weighted perception and the principal component analysis method.

\footnotetext{
1 Docente de la Universidad de Tecnológica Empresarial de Guayaquil. Ecuador

2 Decano de la Facultad de Posgrado Universidad Tecnológica Empresarial de Guayaquil. Ecuador
} 


\section{Jornada de Investigación Universidad Tecnológica Empresarial de Guayaquil}

Keywords: ecosystem, entrepreneur, segmentation, non-experimental, cross, correlational

\section{Introducción}

El emprendimiento es concebido como motor del desarrollo del país y pieza en la transformación de la matriz productiva. Al seguir esta línea se escoge el turismo por ser una de las industrias priorizadas en los planes nacionales del Ecuador, además de ser reconocido como uno de los ejes que impulsa el desarrollo económico en los países emergentes (Liu \& Wall, 2006).

El desarrollo económico y social de una región puede estar sustentado en el fomento de ecosistemas emprendedores innovadores que creen productos y servicios de largo plazo que consoliden la empresa y le permita crecer, sin embargo, para el éxito de ese ecosistema se deben considerar algunos factores como: las políticas públicas, mercados, transferencia de tecnología, innovación, normas culturales y sociales, entre otros (ProChile, 2014).

En el caso específico de América Latina, Hidalgo, Kamiya \& Reyes (2014), reportan que a pesar de los esfuerzos realizados por los países de esta región de conformar ecosistemas emprendedores los resultados no han sido siempre exitosos y algunos de los factores que inciden en esos resultados son los institucionales.

La Teoría Institucional provee un marco conceptual y metodológico que es propicio para abordar esos estudios de los ecosistemas emprendedores, la cual fundamentada desde las ciencias económicas, políticas, psicológicas y sociológicas conciben su marco teórico y metodológico donde las organizaciones deben ser isomorfas al entorno (Rutherford, 1995).

Desde la Teoría Institucional se conciben reglas del juego del entorno institucional que condicionan la actividad emprendedora (North, 1993), que Scott (1995) distingue en regulatorios, normativos y cognitivos donde se articulan desde las políticas institucionales, hasta las normas culturales, pasando por el conocimiento y habilidades de los emprendedores. Esos factores moderan el accionar de las organizaciones y los comportamientos de los individuos dentro de las mismas.

ProChile (2014) parte del hecho que el ecosistema emprendedor debe considerarse innovador, donde se incluyan todas las organizaciones y actores que se interrelacionan dinámicamente para generar innovación, lo que conlleva a crear empleos e ingresos de otras fuentes. En ese tipo de ecosistema el papel fundamental lo lleva el talento humano siempre que tenga la intención y el comportamiento innovador y de generar ideas.

La Teoría Institucional aborda el entorno donde están inmersos los emprendimientos, entorno que está determinado por los valores, normas o creencias de sus actores que inciden en las organizaciones y moderan su comportamiento (Meyer \& Rowan, 1977). Las organizaciones serán de éxito si son capaces de adaptarse a las reglas y normas institucionales impuestas por el entorno (DiMaggio \& Powell, 1983). 


\section{Jornada de Investigación Universidad Tecnológica Empresarial de Guayaquil}

Venkataraman (1997) describe que el proceso de emprendimiento inicia con el descubrimiento y evaluación de las oportunidades del mercado o de la tecnología para crear nuevos productos. Abordar cómo ocurre ese proceso es parte de los factores de la intención emprendedora del ecosistema, se buscan los motivos, el momento y la forma de hacerlo centrado en el emprendedor.

El emprendedor como ápice de la intención emprendedora es visto como una persona con cualidades únicas y es tratado desde las más diversas perspectivas. La escuela psicológica ve al emprendedor como individuo portador de virtudes, valores, comportamientos que lo impulsan a la autorrealización o a asumir riesgos.

La orientación emprendedora es un término que se estableció en la década de los 90 del siglo pasado, a partir de las ideas de Schumpeter (1934) en su libro "Teoría del Desenvolvimiento Económico", donde se da la idea que emprender es combinar los medios de producción para hacer las cosas de manera distinta. Idea que sirve de punto de quiebre para valorar si una empresa está orientada a emprender o no, dada la relación de emprender con la oportunidad para crecer e innovar (Brush \& Chaganti, 1998).

En el comportamiento emprendedor se pueden distinguir dos categorías: el comportamiento emprendedor para crear una nueva empresa (Low \& McMillan, 1988) y emprendimiento desde las habilidades personales para distinguir y aprovechar una oportunidad de mercado (Stevenson \& Gumpert, 1985). La intención emprendedora genera el comportamiento emprendedor (Fishbein \& Aizen, 1975). El comportamiento emprendedor se refiere al desempeño del emprendedor dada sus necesidades, conocimientos, creencias y valores.

La legitimidad surge de las relaciones de la organización con sus grupos de intereses como los clientes, proveedores, competidores, medios de comunicación, las instituciones y los ciudadanos en general. Es un estatus que confieren los grupos de intereses, luego un emprendimiento será legítimo si es isomorfo a los valores y expectativas de los actores sociales (Ashford \& Gibbs, 1990).

La reputación posee un carácter social y se da a través de un proceso de legitimización en el tiempo que es resultado de los continuos ajustes de la empresa al entorno institucional, tanto en las relaciones hacia dentro de la organización, como en las relaciones hacia afuera de la organización, así la reputación se convierte en un activo intangible de la empresa (Rao, 1994).

La industria hotelera ha ido al ritmo de los avances de las nuevas tecnologías y las necesidades de sus clientes que si bien en un principio eran básicas: comida y techo, hoy son mucho más complejas al tender hacia la autorrealización y el ego personal. El hotel es un servicio que crea empleo y con eficiencia genera ganancias apropiadas y es elemento esencial en el viaje turístico, al brindar los servicios complementarios para que el visitante disfrute de los atractivos turísticos del lugar, que en ocasiones es el propio hotel.

Mestre (1999, p.1) aporta su definición de hotel al considerarlo como "todo aquel establecimiento que se dedica profesionalmente y habitualmente a proporcionar 


\section{Jornada de Investigación Universidad Tecnológica Empresarial de Guayaquil}

alojamiento a las personas, mediante precios, con o sin servicios de carácter complementario".

El objetivo que sustenta esta investigación induce que las medidas de las variables en estudio deben realizarse de forma transversal, así la captación de datos se realizó entre diciembre de 2014 y enero de 2015.

\section{Metodología}

Se empleó un diseño no-experimental transeccional correlacional, a partir de encuestar a los emprendedores una vez en el tiempo y sobre esas respuestas tratar de estructurar las relaciones de las variables, así como los agrupamientos de los respondientes en función de las variables que más aporten a la explicación del fenómeno.

Se consideraron 947 instalaciones hoteleras bajo los siguientes criterios: Criterios de inclusión:

* Hoteles de la ciudad de Guayaquil con categorías de tres, cuatro y cinco estrellas.

* Hoteles de la ciudad de Guayaquil que se encuentren en el Consolidado Nacional de Turismo del 2003 y se complementan con las referencias de TripAdvisor.

Criterios de exclusión:

* No se pueda aplicar la encuesta por no acceder a la instalación.

* Negativa de los emprendedores a llenar la encuesta.

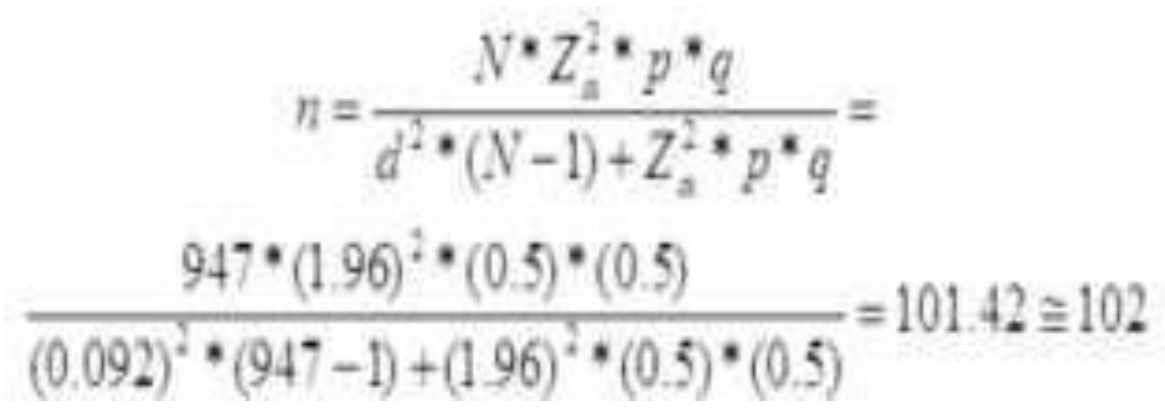

En total se tomaron 102 encuestas en 34 emprendimientos hoteleros y en función de la cantidad de empleados se decidía a cuántos entrevistar. Los criterios de encuestado estaban dados por:

* Fundador del emprendimiento.

* Desarrolle roles de toma de decisiones estratégicas en el emprendimiento.

* Sea un inter emprendedor en la empresa. 
Revista Ciencia \& Tecnología No. 16, 31 de octubre de 2017 ISSN impreso: 1390 - 6321

\section{Jornada de Investigación Universidad Tecnológica Empresarial de Guayaquil}

Tabla 1: Criterio de muestreo en el estudio del sector hotelero de Guayaquil

\begin{tabular}{|c|c|}
\hline Sector & Hoteles de Guayaquil de 3, 4 y 5 estrellas \\
\hline $\begin{array}{l}\text { Ubicación } \\
\text { geográfica }\end{array}$ & Guayas, Guayaquil \\
\hline Metodologia & Cuestionario estructurado \\
\hline $\begin{array}{l}\text { Procedimiento } \\
\text { de muestreo }\end{array}$ & Muestreo aleatorio de poblaciones finitas \\
\hline $\begin{array}{c}\text { Población de } \\
\text { estudia }\end{array}$ & Emprendedores del sector hotelero \\
\hline $\begin{array}{c}\text { Tamano de la } \\
\text { muestra }\end{array}$ & 102 respondientes \\
\hline $\begin{array}{l}\text { Tasa de } \\
\text { respuesta }\end{array}$ & $98.03 \%$ \\
\hline Ertor muestral & $0.092 \%$ \\
\hline $\begin{array}{l}\text { Nivel de } \\
\text { confianza }\end{array}$ & $95 \%, p=q=0.5, Z=1.96$ \\
\hline $\begin{array}{l}\text { Período de } \\
\text { recogida de } \\
\text { datos }\end{array}$ & Diciembre/2014 y Enera/2015 \\
\hline
\end{tabular}

Fuente: Elaboración propia

Los encuestados por emprendimientos oscilan entre uno a cuatro en función de la cantidad de empleados.

Tabla 2: Operacionalización de las variables de estudio y reactivos empleados en la encuesta realizada

\begin{tabular}{|c|c|}
\hline \multicolumn{2}{|r|}{ Factores Institucionales } \\
\hline \multirow{5}{*}{ Regulador } & $\begin{array}{l}\text { 1. Las instituciones públicas en este país ayudan a los } \\
\text { emprendedores a realizar nuevas actividades empresariales }\end{array}$ \\
\hline & $\begin{array}{l}\text { 2. Los organismos gubernamentales ofrecen fondos públicos } \\
\text { suficientes para nuevas actividades de negocio de los } \\
\text { emprendedores }\end{array}$ \\
\hline & $\begin{array}{l}\text { 3. Las instituciones nacionales y locales apoyan preferentemente } \\
\text { los emprendedores que quieren realizar nuevas actividades } \\
\text { empresariales }\end{array}$ \\
\hline & $\begin{array}{l}\text { 4. Incluso, después de fracasar en la actividad emprendedora, las } \\
\text { instituciones estimulan a los emprendedores que intenten poner en } \\
\text { marcha nuevas actividades empresariales }\end{array}$ \\
\hline & $\begin{array}{l}\text { 5. Los emprendedores saben cómo proteger legalmente una nueva } \\
\text { empresa }\end{array}$ \\
\hline Cognitivo & 6. Los emprendedores saben cómo gestionar el riesgo \\
\hline
\end{tabular}




\section{Jornada de Investigación Universidad Tecnológica Empresarial de Guayaquil}

\begin{tabular}{|c|c|}
\hline & $\begin{array}{l}\text { 7. Los emprendedores saben dónde encontrar información para el } \\
\text { desarrollo de nuevas actividades empresariales }\end{array}$ \\
\hline \multirow[t]{3}{*}{ Normativo } & $\begin{array}{l}\text { 8. En este país, el pensamiento innovador y creativo es visto como } \\
\text { una ruta hacia el éxito }\end{array}$ \\
\hline & 9. Los emprendedores son personas admiradas en nuestro país \\
\hline & $\begin{array}{l}\text { 10. La sociedad admira a aquellas personas que son } \\
\text { emprendedoras }\end{array}$ \\
\hline \multicolumn{2}{|c|}{ Orientación Emprendedora } \\
\hline \multirow{2}{*}{$\begin{array}{l}\text { Asunción de } \\
\text { Riesgo }\end{array}$} & 11. Los emprendedores asumen el riesgo de fracasar \\
\hline & $\begin{array}{l}\text { 12. Los emprendedores actúan con valentía y atrevimiento para } \\
\text { lograr sus objetivos }\end{array}$ \\
\hline Proactividad & $\begin{array}{l}\text { 13. Los emprendedores suelen tomar la iniciativa en las } \\
\text { negociaciones e introducen nuevas técnicas y herramientas en su } \\
\text { gestión administrativa }\end{array}$ \\
\hline \multirow[t]{2}{*}{ Innovación } & $\begin{array}{l}\text { 15. Los emprendedores introducen nuevos productos y/o servicios } \\
\text { frecuentemente }\end{array}$ \\
\hline & $\begin{array}{l}\text { 16. Los emprendedores hacen investigación e innovación en sus } \\
\text { empresas }\end{array}$ \\
\hline \multirow[t]{2}{*}{ Autonomía } & $\begin{array}{l}\text { 17. Los emprendedores permiten que sus empleados tengan } \\
\text { iniciativas propias }\end{array}$ \\
\hline & $\begin{array}{l}\text { 18. Los emprendedores permiten que sus empleados } \\
\text { busquen oportunidades de negocio sin tener que } \\
\text { constantemente hacerle consultas }\end{array}$ \\
\hline \multirow[t]{2}{*}{$\begin{array}{l}\text { Agresividad } \\
\text { competitiva }\end{array}$} & $\begin{array}{l}\text { 19. Los emprendedores desafían directa e indirectamente a sus } \\
\text { competidores en el mercado }\end{array}$ \\
\hline & $\begin{array}{l}\text { 20. Los emprendedores buscan tener una mejor posición en el } \\
\text { mercado }\end{array}$ \\
\hline \multicolumn{2}{|c|}{ Intención Emprendedora } \\
\hline \multirow{4}{*}{$\begin{array}{l}\text { Actitud hacia } \\
\text { el riesgo }\end{array}$} & 21. Ser emprendedor y tener mi propia empresa \\
\hline & me reportaría más ventajas que inconvenientes \\
\hline & 22. La carrera de empresario/a me resulta atractiva \\
\hline & 23. Ser un empresario es una gran satisfacción \\
\hline \multirow{3}{*}{$\begin{array}{l}\text { Norma } \\
\text { subjetiva }\end{array}$} & 24. Mis amigos aprueban mi decisión de crear una empresa \\
\hline & $\begin{array}{l}\text { 25. Mi familia más directa aprueba mi decisión de crear una } \\
\text { empresa }\end{array}$ \\
\hline & 26. Mis colegas aprueban mi decisión de crear una empresa \\
\hline \multirow[t]{4}{*}{ Auto eficacia } & $\begin{array}{l}\text { 27. Me siento capaz de reconocer el potencial de una idea u } \\
\text { oportunidad, que permitan el posicionamiento o la ampliación de } \\
\text { mi empresa }\end{array}$ \\
\hline & $\begin{array}{l}\text { 28. Me siento capaz de descubrir nuevas formas para mejorar los } \\
\text { productos o servicios existentes }\end{array}$ \\
\hline & $\begin{array}{l}\text { 29. Me siento capaz de identificar nuevas áreas de crecimiento } \\
\text { potencial y nichos de mercado rentables para un producto o } \\
\text { servicio }\end{array}$ \\
\hline & $\begin{array}{l}\text { 30. Me siento capaz de crear productos o servicios para } \\
\text { necesidades no satisfechas de consumidores }\end{array}$ \\
\hline
\end{tabular}




\section{Jornada de Investigación Universidad Tecnológica Empresarial de Guayaquil}

Intención emprendedora

Creatividad
Experiencia
Descubridora

Legitimidad

Experiencia integradora

31. Identifiqué una oportunidad y establecí mi propia empresa

32. He desarrollado nuevos productos y/o servicios en mi empresa

33. Tengo conformado un equipo de trabajo en mi empresa

34. Tengo elaborado un plan de negocio de mi empresa

35. He asistido a cursos, seminarios y talleres sobre emprendimiento

\section{Comportamiento Emprendedor}

36. Disfruto pensar o visualizar en nuevas oportunidades

37. Usualmente puedo percibir las nuevas oportunidades

38. Me describo a mí mismo como una persona que aprovecha las oportunidades

39. Regularmente para identificar buenas oportunidades tengo que profundizar en el mercado

40. Es importante que cuando identifique la nueva oportunidad sea un concepto que pueda ser desarrollado con tiempo suficiente

41. Las nuevas oportunidades generalmente provienen del mercado o de cambios tecnológicos

42. La retroalimentación de los clientes, permite hacer grandes cambios de las nuevas oportunidades

43. Mis nuevas oportunidades son tecnológicamente factibles de poder realizarse

44. Lo más importante es creer que la nueva oportunidad se puede realizar

\begin{tabular}{|l|l|}
\hline \multirow{5}{*}{ Corrección } & $\begin{array}{l}\text { 45. Todas las actividades que desarrolla la organización ayudan a } \\
\text { que ésta pueda alcanzar sus objetivos }\end{array}$ \\
\cline { 2 - 3 } $\begin{array}{l}\text { 46. Todas las actividades que desarrolla la organización «se deben } \\
\text { hacer» con independencia de su utilidad para conseguir los } \\
\text { objetivos }\end{array}$ \\
\cline { 2 - 2 } $\begin{array}{l}\text { 47. La organización desarrolla actividades que ayudan a simplificar } \\
\text { los procesos de decisión, consiguiendo que las decisiones sean } \\
\text { mejores y más racionales }\end{array}$ \\
\hline Respaldo & $\begin{array}{l}\text { 48. Los compañeros creen que todas las actividades que desarrolla } \\
\text { la organización ayudan a que ésta pueda alcanzar sus objetivos }\end{array}$ \\
\cline { 2 - 2 } & $\begin{array}{l}\text { 49. Los compañeros creen que todas las actividades que } \\
\text { desarrolla la organización «se deben hacer» con independencia de } \\
\text { su utilidad para conseguir los objetivos organizativos }\end{array}$ \\
\cline { 2 - 2 } $\begin{array}{l}\text { 50. Los compañeros creen que su organización desarrolla } \\
\text { actividades que ayudan a simplificar los procesos de decisión, } \\
\text { consiguiendo que las decisiones sean mejores y más racionales }\end{array}$ \\
\hline $\begin{array}{l}\text { 51. Su(s) jefe(s) creen que todas las actividades que desarrolla la } \\
\text { organización ayudan a que ésta pueda alcanzar sus objetivos }\end{array}$ \\
\hline $\begin{array}{l}\text { 52. Su(s) jefe(s) creen que todas las actividades que desarrolla la } \\
\text { organización «se deben hacer» con independencia de su utilidad } \\
\text { para conseguir los objetivos organizativos }\end{array}$ \\
\hline
\end{tabular}




\section{Jornada de Investigación Universidad Tecnológica Empresarial de Guayaquil}

\begin{tabular}{|l|l|} 
& 53. Su(s) jefe(s) creen que su organización \\
\hline & $\begin{array}{l}\text { desarrolla actividades que ayudan a simplificar los } \\
\text { procesos de decisión, consiguiendo que las decisiones sean } \\
\text { mejores y más racionales }\end{array}$ \\
\hline Reputación & \begin{tabular}{l} 
54. Los emprendimientos son apoyados por la sociedad \\
\cline { 2 - 3 } \\
\cline { 2 - 2 }
\end{tabular} 55. Ser emprendedor es parte de la cultura de este país \\
\hline
\end{tabular}

Fuente: Elaboración propia

Para la validación de la escala de medida se emplea la encuesta como la técnica de colección de datos donde se empleó la escala de Likert de siete opciones de respuesta para poder captar la mayor diversidad posible para obtener resultados óptimos cuando se empleen las técnicas estadísticas.

Tabla 3: Validación de la escala de medida

\begin{tabular}{|c|c|c|c|c|c|c|}
\hline $\begin{array}{c}\text { Totalme } \\
\text { nte de } \\
\text { acuerdo }\end{array}$ & $\begin{array}{l}\text { Muy } \\
\text { de } \\
\text { acuer } \\
\text { do }\end{array}$ & $\begin{array}{c}\text { De } \\
\text { acuerd } \\
0\end{array}$ & $\begin{array}{c}\text { Ni de } \\
\text { acuerdo } \\
\text { ni } \\
\text { en } \\
\text { desacue } \\
\text { rdo }\end{array}$ & $\begin{array}{c}\text { En } \\
\text { desacue } \\
\text { rdo }\end{array}$ & $\begin{array}{l}\text { Muy en } \\
\text { desacue } \\
\text { rdo }\end{array}$ & $\begin{array}{c}\text { Totalme } \\
\text { nte en } \\
\text { desacuer } \\
\text { do }\end{array}$ \\
\hline 7 & 6 & 5 & 4 & 3 & 2 & 1 \\
\hline
\end{tabular}

Fuente: Elaboración propia

Se valoraron las cualidades de la técnica. En el caso de la fiabilidad como la repetición de la medida en similares circunstancias se empleó el Alfa de Cronbach, el que reportó un valor de 0.9616 que resulta una alta consistencia interna.

Mientras que la validez se induce a partir del KMO y la prueba de esfericidad de Bartlett que reportan un valor de KMO de 0.777 y una $c 2=4958.467(p=0.000)$ lo que resulta una alta interrelación entre las variables, pero la matriz de correlación no es idéntica. Así la escala empleada en esta investigación es fiable y válida.

Medida de Kaiser Meyer Olkin (KMO) y prueba de esfericidad de Bartlett resultante del análisis de reducción de dimensiones. 


\section{Jornada de Investigación Universidad Tecnológica Empresarial de Guayaquil}

\section{Tabla 4: Prueba de KMO y Bartlett}

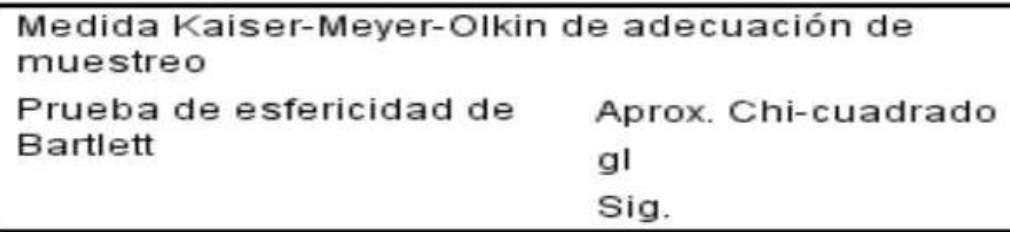

Fuente: Salida del SPSS (v.22, 2013)

En la aplicación de la encuesta se trató de crear la motivación y confianza en su llenado, se hizo de manera individual y de forma que primara una atmósfera adecuada para el respondiente. Siempre se logró el compromiso por parte de la gerencia de la instalación, y se fue proactivo en caso que se notara influencias de otras personas en el llenado.

La tabulación fue validada por muestreo al azar de encuestas y el contraste de sus datos correspondientes en la matriz de datos elaborada inicialmente en Excel y luego pasada al STATISTICA (v. 10.0; 2011).

1.- Se comienza por un análisis de la representatividad de la muestra al considerar la pertenencia de emprendedores y emprendimientos a las categorías de clasificación que se contemplan en la encuesta.

2.- En el caso de los emprendedores se analizó las frecuencias de respondientes en cuanto al sexo, grupo etario y nivel educacional. Mientras que relativo al emprendimiento se refieren el tiempo de establecido el mismo, la etapa de desarrollo en la cual percibe el respondiente, cantidad de empleados e ingresos anuales.

3.- Luego, se valora el índice ponderado de percepción del respondiente. El índice ponderado de las percepciones sobre los factores del ecosistema emprendedor se calcula de la siguiente manera:

4.- Con el empleo del STATISTICA se realizaron los análisis que fundamentan los resultados expuestos, para ello se usó el análisis de componentes principales para identificar la estructura que subyace a las variables que se estudian, así como para configurar el plano factorial del primer y segundo eje (Hair et al. 1999).

5.-Una vez identificado los respondientes que integran cada grupo se procedió a determinar sus características comunes en cuanto a sus propias tipologías como las del emprendimiento a los cuales representan.

6.- Antes de realizar al análisis de componentes principales se realizó la inspección de la matriz de datos. Primero, se buscaron datos fuera de rangos de los cuales no se encontraron ninguno gracias a la validación de la hoja de cálculo de Excel empleada para tal fin.

7.- Luego, se buscó datos perdidos que en este caso estaban presentes en tres 


\section{Jornada de Investigación Universidad Tecnológica Empresarial de Guayaquil}

respondientes. También, se realizaron exploraciones de las correlaciones entre algunas variables de interés, así como el cálculo del KMO y la prueba de esfericidad de Bartlett como antecedentes del análisis de componentes principales.

La validación de los resultados del análisis de componentes principales se realizó al dividir la muestra en dos partes, a partir de la selección aleatoria de los respondientes que integrarían cada una de las muestras. A esas dos muestras se aplicó el análisis de componentes principales y se encontró en general resultados similares en ambos análisis.

\section{Resultados y discusión}

Caracterización de los emprendedores encuestados En la figura 1 se muestra la frecuencia en cuanto al sexo de los emprendedores encuestados, el $51 \%$ es masculino y el $49 \%$ femenino. Mientras que en relación a la edad se tiene que los grupos más representados son los de 25 a 34 años y de 35 a 45 años con un $45 \%$ y $42 \%$, respectivamente (figura 2 ).

Existe una representatividad adecuada en cuanto a sexo y edad si se considera que las edades de 25 a 45 años es la etapa de mayor protagonismo laboral de las personas.

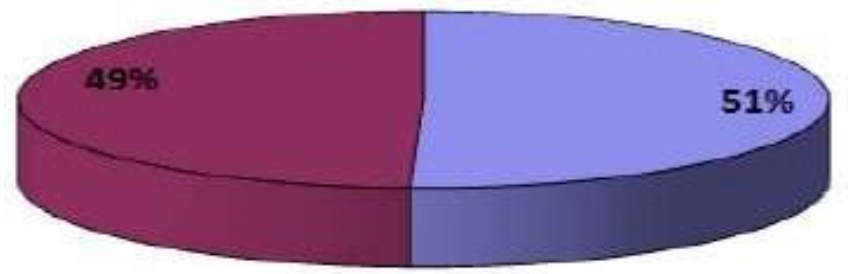

口Masculino DFemenino

Gráfico 1: Frecuencia de correspondientes en cuanto al sexo

Fuente: Elaboración propia

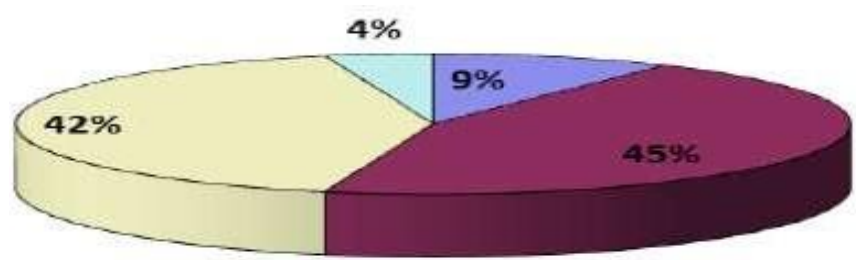

$\square 18$ a $24 \quad \square 25$ a 34

Gráfico 2: Frecuencia de respondientes en cuanto a la edad

Fuente: Elaboración propia 


\section{Jornada de Investigación Universidad Tecnológica Empresarial de Guayaquil}

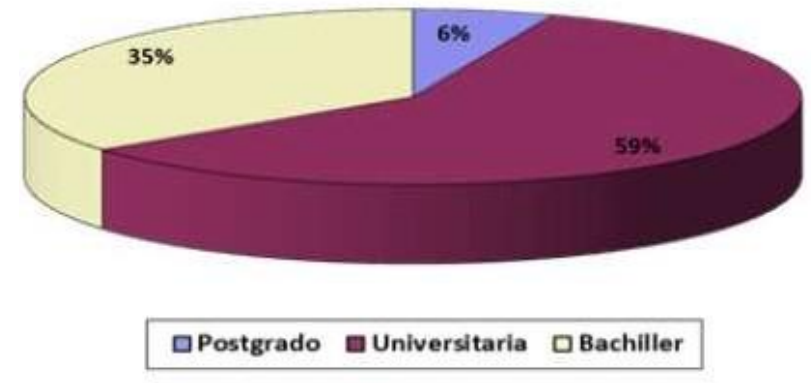

Gráfico 3: Frecuencia de respondientes en cuanto a estudios terminados Fuente: Elaboración propia

En cuanto al nivel escolar terminado la figura 3 nos muestra que el $6 \%$ poseen postgrados, el $59 \%$ son universitarios y el $35 \%$ bachiller. Devela que los emprendimientos en el sector hotelero requieren de preparación técnica para la gestión del emprendimiento.

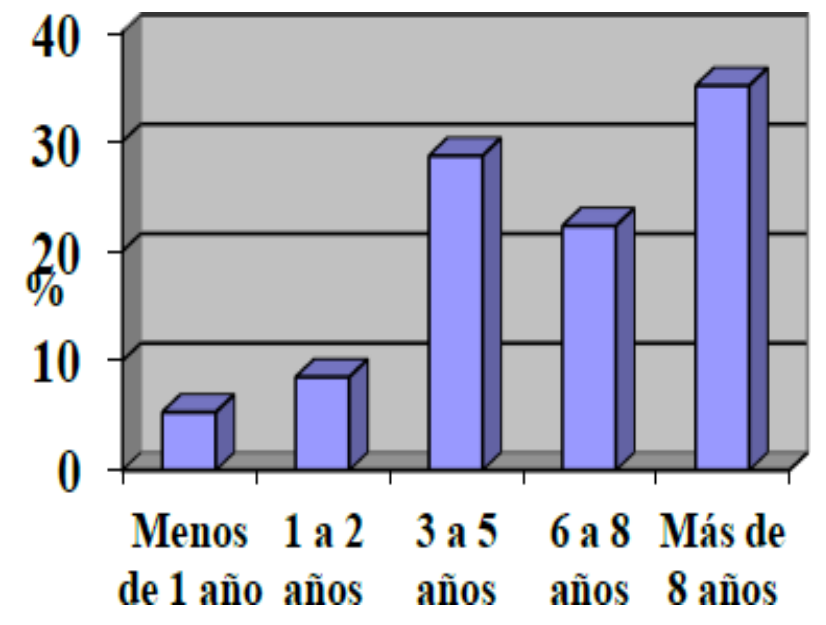

Gráfico 4: Frecuencia de respondientes en cuanto al tiempo de establecido el emprendimiento

Fuente: Elaboración propia

Por otra parte, la figura 4 ilustra la frecuencia de tiempo de establecido el emprendimiento, donde se logró captar información de todas las categorías estipuladas. Dada las características del sector hotelero es de esperar que los emprendimientos posean algún tiempo de establecido. 


\section{Jornada de Investigación Universidad Tecnológica Empresarial de Guayaquil}

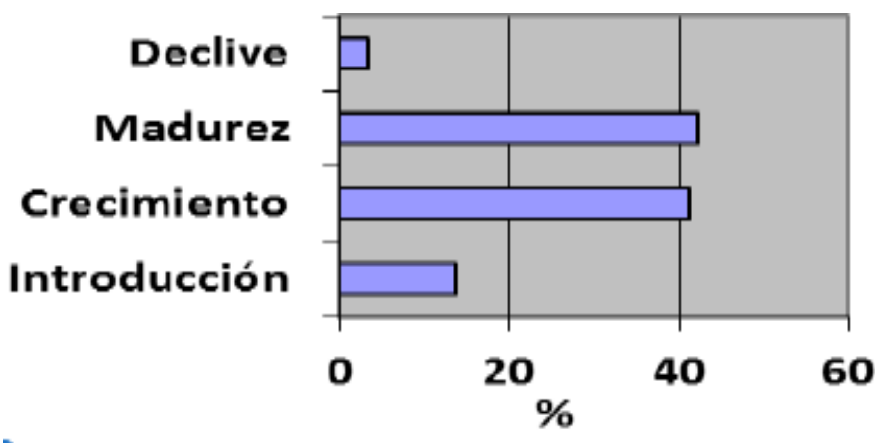

Gráfico 5: Frecuencia de respondientes en cuanto al estadio de desarrollo del emprendimiento

Fuente: Elaboración propia

En cuanto al estadio de desarrollo que consideran los respondientes de los emprendimientos existe representación de todas las categorías dispuestas, donde las mayores frecuencias pertenecen a la fase de crecimiento $(41.1 \%)$ y madurez $(42.1$ $\%$ ). Mientras que en declive se consideran el 3.2\% de los emprendedores encuestados (figura 5).

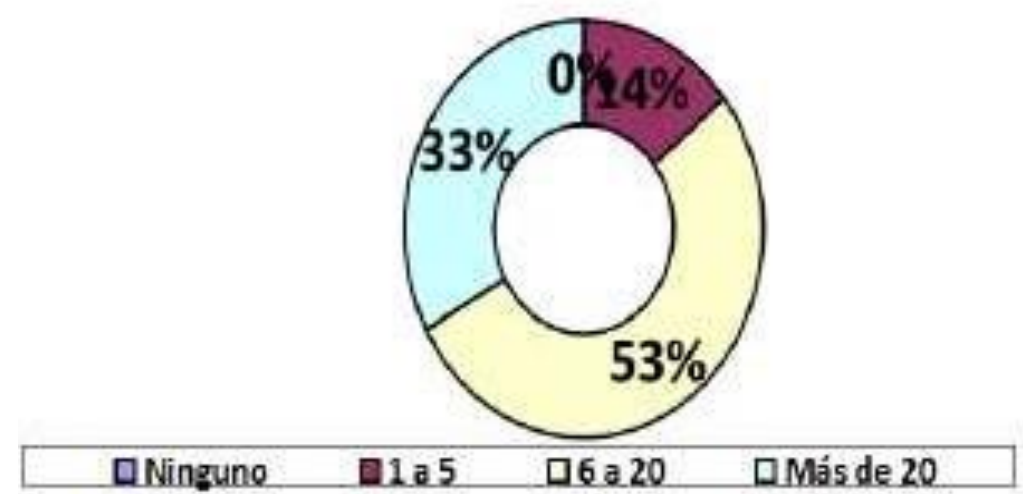

\section{Gráfico 6: Frecuencia de respondientes en cuanto a la cantidad de empleados}

Fuente: Elaboración propia

En cuanto a la cantidad de empleados la mayor frecuencia se ubica en emprendimientos que emplean de 6 a 20 personas con el $53 \%$, mientras que más de 20 empleados son el 33\% de los emprendimientos. No existe caso sin ningún empleado y dada las características del sector hotelero es poco probable que exista (figura 6). 
Revista Ciencia \& Tecnología No. 16, 31 de octubre de 2017 ISSN impreso: 1390 - 6321

\section{Jornada de Investigación Universidad Tecnológica Empresarial de Guayaquil}

En la figura 7 se exponen las frecuencias observadas de los emprendimientos en cuanto al nivel de ingresos anuales, la mayor concentración de establecimientos hoteleros está en más de 72001.00 dólares al año. Sin embargo, existen emprendimientos en la muestra de niveles inferiores de ingresos anuales, excepto para la más baja de las categorías, menos de 2880.00 dólares al año. Los datos presentados y los comentarios realizados patentizan que la muestra tomada para el estudio recorre la diversidad de características de los emprendedores y del propio emprendimiento que validan la muestra. La representatividad de la muestra en la diversidad de situaciones es el punto de partida para explorar el ecosistema emprendedor de un sector.

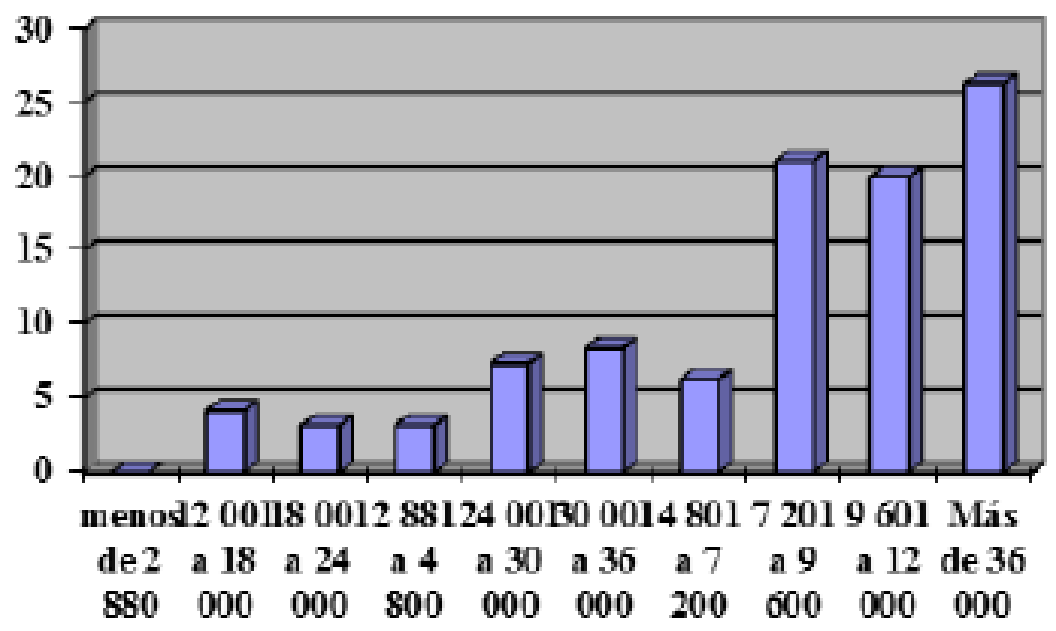

\title{
Gráfico 7: Frecuencia de respondientes en cuanto a los ingresos anuales que genera el emprendimiento
}

\author{
Fuente: Elaboración propia
}

Índice ponderado de la percepción de los emprendedores de los factores que componen el ecosistema emprendedor del sector hotelero de Guayaquil.

En la figura 8, se muestra el índice ponderado de percepción de las dimensiones del ecosistema del sector hotelero, donde se puede observar que el valor más bajo pertenece a los factores institucionales, mientras que los más altos índices se reportan en intención emprendedora y comportamiento emprendedor. Se debe hacer notar, que aunque se reportan en general índices ponderados que se pueden calificar de buenos, existe una brecha prácticamente de un punto o más por llegar al valor ideal de siete. 


\section{Jornada de Investigación Universidad Tecnológica Empresarial de Guayaquil}
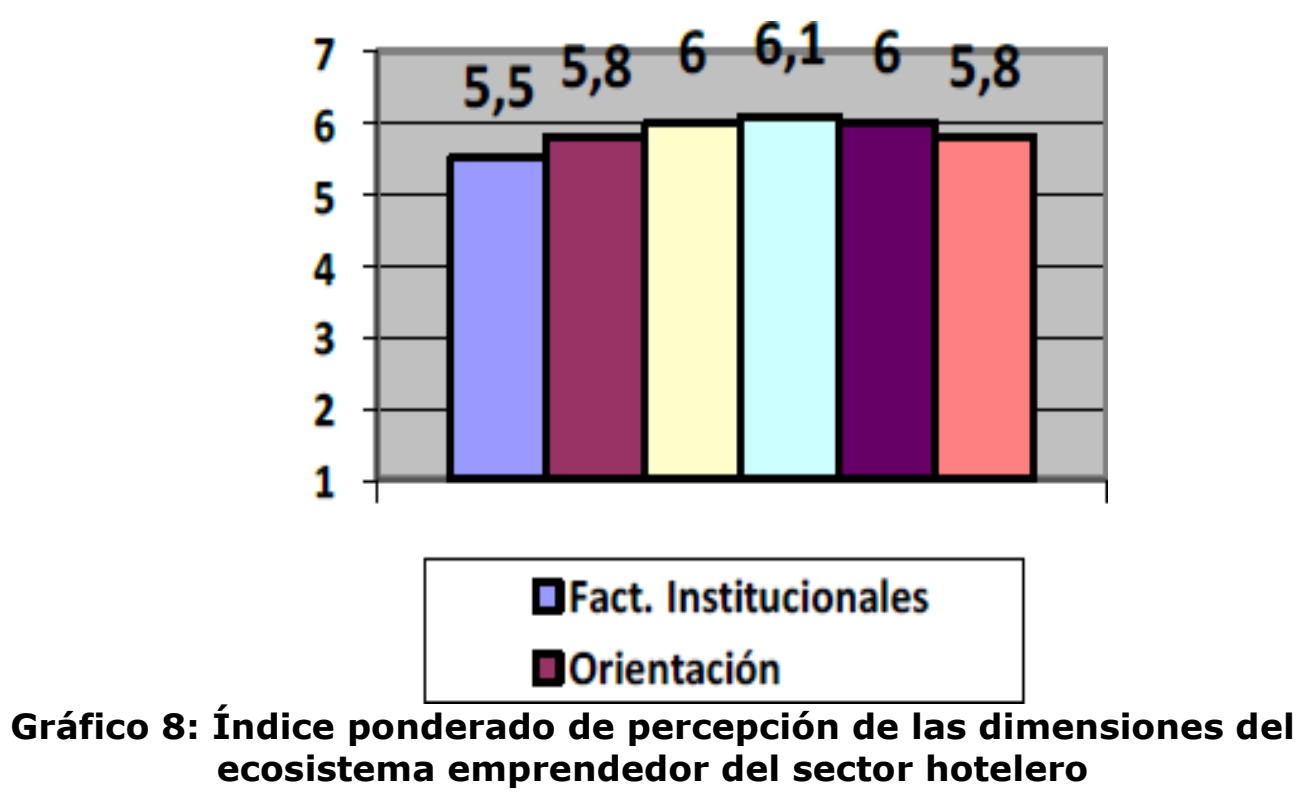

Fuente: Elaboración propia

La figura 9 muestra el índice ponderado de los factores institucionales, se puede observar que el menor valor reportado es en la pregunta 4, que de hecho es el más bajo reportado en todos los reactivos. Los demás reactivos oscilan entre 5.3 y 5.9 , Ilama la atención que en ningún caso alcanza el valor de seis. Los reactivos p2, p3, p4, están por debajo del índice ponderado de los factores institucionales, los que pertenecen al factor regulador.

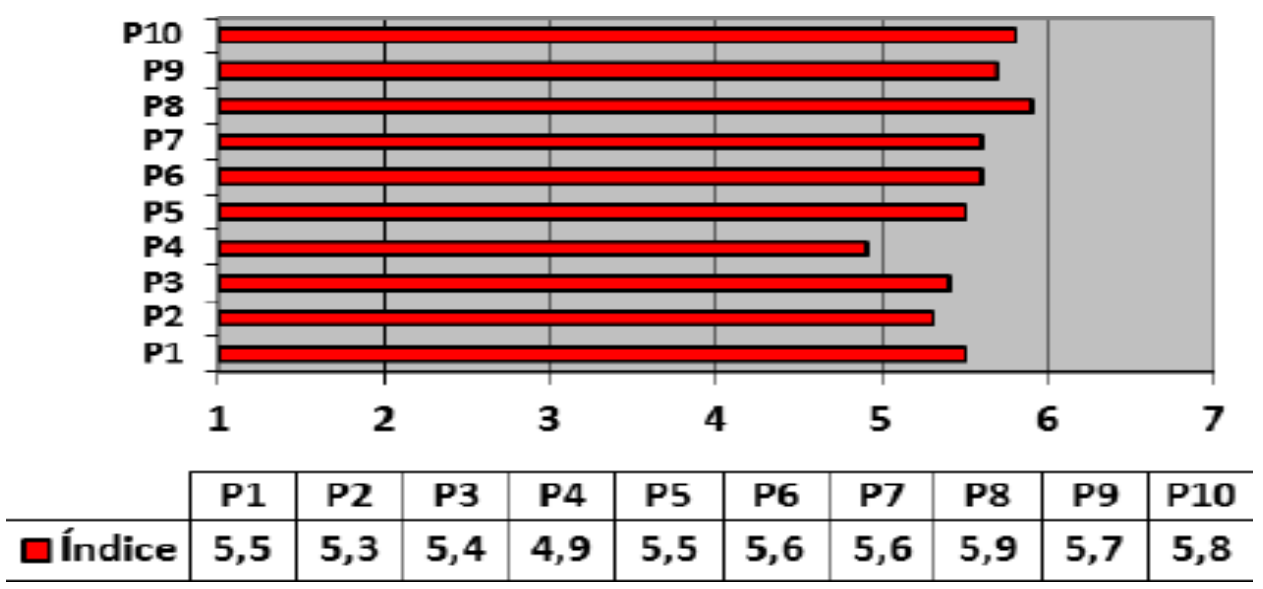

Gráfico 9: Índice ponderado de los factores institucionales reportados en el ecosistema emprendedor del sector hotelero

Fuente: Elaboración propia 


\section{Jornada de Investigación Universidad Tecnológica Empresarial de Guayaquil}

En la figura 10 referida a la orientación emprendedora los reactivos p17 y p18 están por debajo del índice reportado para la orientación emprendedora.

Esos reactivos pertenecen a la autonomía. Mientras que la figura 13 refiere el índice ponderado de la intención emprendedora y presenta los menores valores en los reactivos: p24, p26 de la norma subjetiva; p30 de la autoeficacia y p31, p32, p33, p34 y p35 de la intención emprendedora.

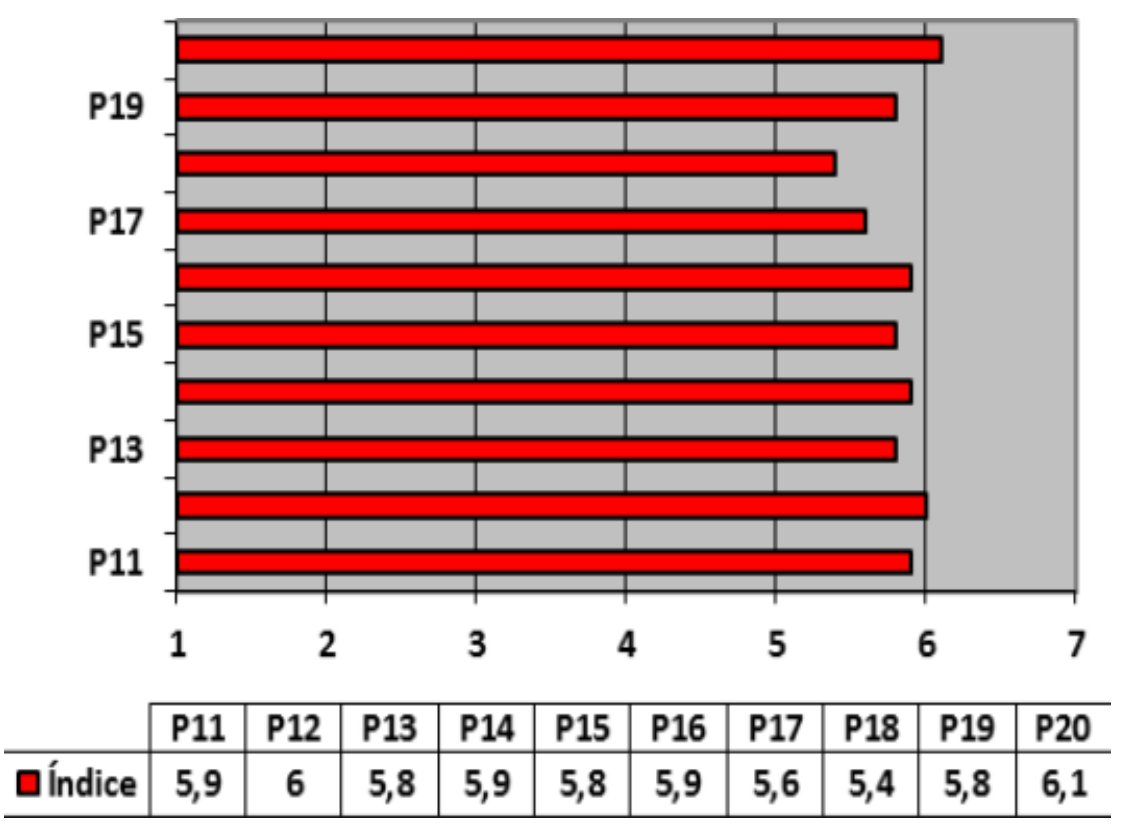

\section{Gráfico 10: Índice ponderado de la orientación emprendedora reportados en el ecosistema emprendedor del sector hotelero}

Fuente: Elaboración propia 


\section{Jornada de Investigación Universidad Tecnológica Empresarial de Guayaquil}



Gráfico 11: Índice ponderado de la intención emprendedora reportados en el ecosistema emprendedor del sector hotelero

Fuente: Elaboración propia

En la figura 12 se muestra que el índice de comportamiento emprendedor presenta sus valores más bajos en los reactivos p36 y p37 propios de la creatividad. Al valorar los índices de legitimidad tiene sus valores más bajos en los reactivos p45 y p46 propios de la corrección, p49 de respaldo y p53 a la autorización (figura 13).

Mientras que los índices ponderados de la reputación se muestran en la figura 14. 


\section{Jornada de Investigación Universidad Tecnológica Empresarial de Guayaquil}

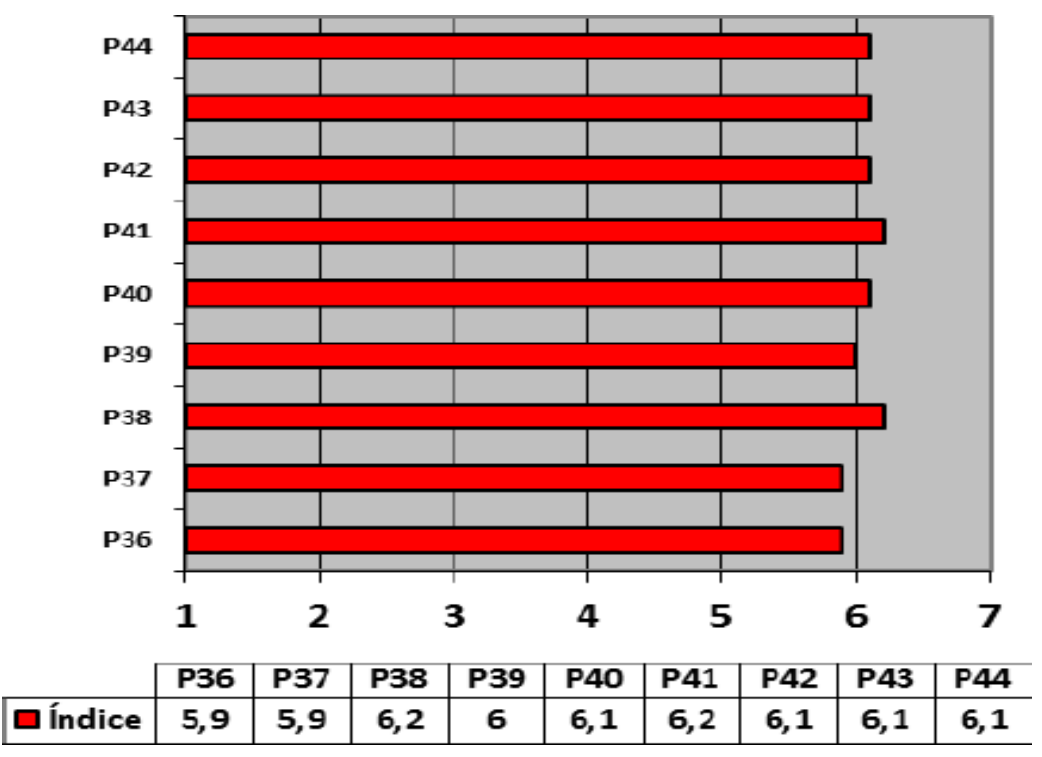

Gráfico 12: Índice ponderado del comportamiento emprendedor reportados en el ecosistema emprendedor del sector hotelero

Fuente: Elaboración propia

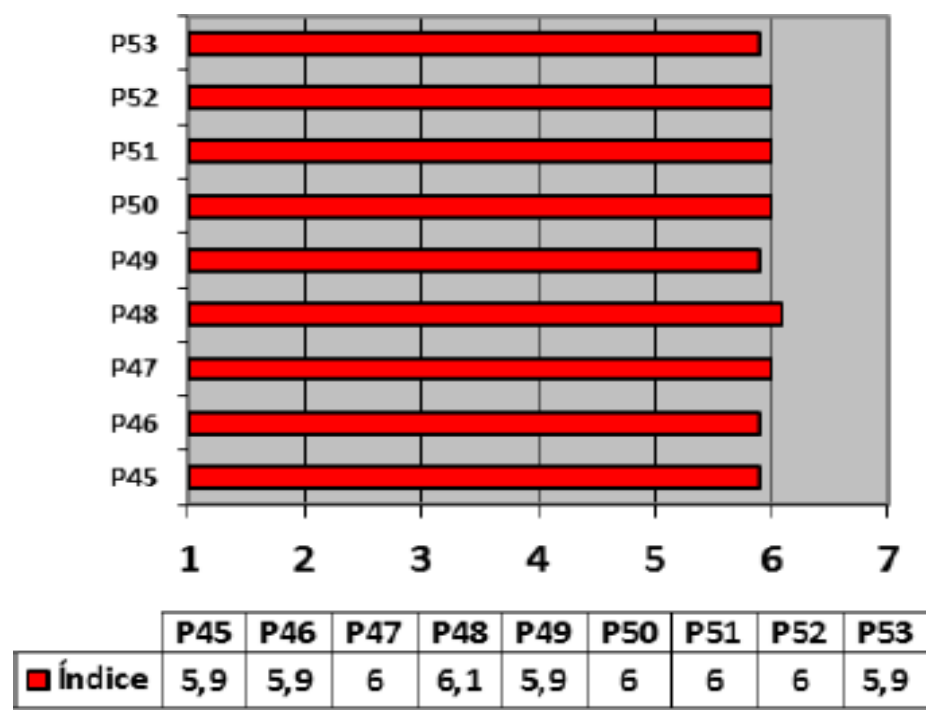

Gráfico 13: Índice ponderado de la legitimidad reportados en el ecosistema emprendedor del sector hotelero

Fuente: Elaboración propia 


\section{Jornada de Investigación Universidad Tecnológica Empresarial de Guayaquil}

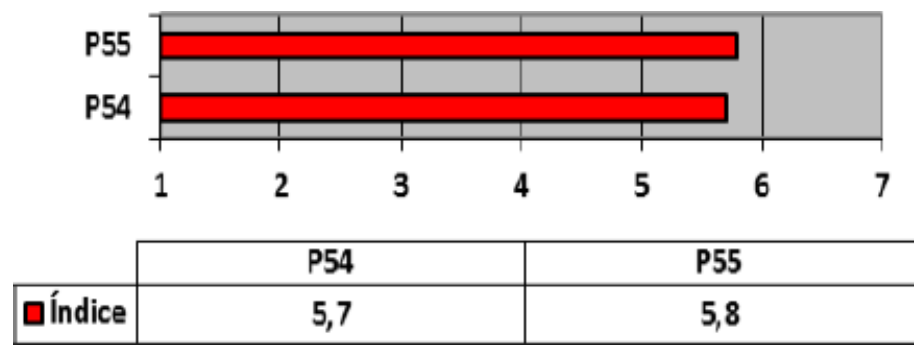

Gráfico 14: Índice ponderado de la reputación reportados en el ecosistema emprendedor del sector hotelero

Fuente: Elaboración propia

Factores que condicionan el ecosistema emprendedor del sector hotelero de Guayaquil

Se realiza un análisis de componentes principales para identificar las estructuras latentes que existen en las relaciones entre los reactivos puestos a consideración de los emprendedores. En ocho ejes se explica el $68.3 \%$ de la varianza total que se considera aceptable para estos tipos de estudios, sobresale el primer eje con una contribución de explicación del $34.0 \%$ de la varianza total, el resto de los ejes tiene explicaciones por debajo del $9 \%$ de la varianza total (tabla 5).

Al valorar la contribución de cada reactivo de la encuesta en la conformación de los ejes, se observa que el primer eje está conformado por la orientación de desafiar la competencia ( 19 ) con la intención de conformar el equipo de trabajo ( $p 33$ ) y poder aprovechar las oportunidades del mercado y la tecnología ( $\mathrm{p} 41$ y $\mathrm{p} 44$ ) y para legitimarse se realizan actividades pertinentes y eficaces en aras de las metas de la organización (p45 y p47).

Luego, el primer eje se centra en elementos de la orientación, intención y comportamiento del emprendedor que condicionan la legitimidad de la organización en cuanto a la relación entre las actividades y las metas organizacionales. 
Revista Ciencia \& Tecnología No. 16, 31 de octubre de 2017 ISSN impreso: 1390 - 6321

\section{Jornada de Investigación Universidad Tecnológica Empresarial de Guayaquil}

Tabla 5: Valores propios y varianza explicada por los ejes resultantes del análisis de componentes principales

\begin{tabular}{|c|c|c|c|}
\hline Ejes & $\begin{array}{l}\text { Valores } \\
\text { propios }\end{array}$ & $\begin{array}{l}\% \\
\text { Varianza }\end{array}$ & $\begin{array}{c}\text { \% } \\
\text { Acumulado }\end{array}$ \\
\hline 1 & 18.71451 & 34.02639 & 34.0264 \\
\hline 2 & 4.74301 & 8.62365 & 42.6500 \\
\hline 3 & 3.79813 & 6.90568 & 49.5557 \\
\hline 4 & 2.83103 & 5.14733 & 54.7031 \\
\hline 5 & 2.25309 & 4.09652 & 58.7996 \\
\hline 6 & 1.94627 & 3.53867 & 62.3383 \\
\hline 7 & 1.66703 & 3.03097 & 65.3692 \\
\hline 8 & 1.59383 & 2.89787 & 68.2671 \\
\hline
\end{tabular}

Fuente: Elaboración propia

En tanto, el segundo eje parte del apoyo institucional a los emprendimientos ( $p 1, p 3$ y p4) y saber gestionar el riesgo y la información como factores institucionales dado por el entorno del ecosistema emprendedor que condicionan la orientación de otorgar autonomía a los empleados en la búsqueda de oportunidades ( 18 ) para visualizar y aprovechar las nuevas oportunidades de negocio (p36 y p38) (tabla 9). Entonces, el segundo eje parte de los factores institucionales dados en el entorno del ecosistema emprendedor, que condicionan la orientación en la autonomía y la visualización y aprovechamiento de las oportunidades como parte de la creatividad del comportamiento emprendedor.

El tercer eje resultante del análisis de componentes principales se determina por algunos de los elementos de la intención emprendedora. Así, la actitud de tener satisfacción de ser empresario (p23) que es aprobado por los amigos y colegas (p24 y p26) como parte de las normas; condiciona la intención de identificar una oportunidad y "establecí" una empresa (p31). Luego, la intención de identificar una oportunidad y establecer una empresa, pasa por elementos sentimentales del emprendedor y la aprobación social de esa toma de decisiones.

El cuarto eje confronta la admiración social de los emprendedores (p10) propio de las normas de los factores institucionales con la asistencia a actividades de formación para emprendedores (p35) perteneciente al comportamiento emprendedor (tabla 9). El quinto componente se basa en la controversia entre la intención de ser capaz de descubrir nuevas formas del producto (p28) e identificar nuevas áreas de crecimiento potencial (p29) con la pertinencia de las actividades con las metas de la organización (p49) de la legitimidad (tabla 9). Mientras que el sexto componente también contrapone la pertinencia de las actividades ( $\mathrm{p48}$ ) de la legitimidad con la intención de elaborar un plan de negocio (p33) y conformar un equipo de trabajo (p34). 


\section{Jornada de Investigación Universidad Tecnológica Empresarial de Guayaquil}

Esas controversias muestran la complejidad del ecosistema emprendedor donde no se deben suponer relaciones simples entre sus componentes. Primero, se describió la contradicción entre el factor institucional normativo de la admiración social de los emprendedores, con el comportamiento de asistir a acciones de formación. Luego, se contraponen la legitimidad de las actividades en aras de las metas de la organización con los elementos de intención emprendedora; por una parte, está la autoeficacia de detectar nuevos productos y áreas potenciales de mercado y; por la otra, con la intención de elaborar plan de negocio y conformar equipo de trabajo.

Igualmente, el séptimo eje se basa en otra contraposición, en este caso de la legitimidad de que las actividades que se desarrollen contribuyan o no a la meta de la organización (p46) con la reputación como parte de la cultura del país (p55).

Por último, el octavo componente se centra sólo en la reputación como acción que es apoyado por la sociedad (p54) (tabla 9). Eso último, indica que la reputación es el éxito que se busca, pero por ser parte de la cultura del país no se debe hacer cualquier tipo de actividades.

Al realizar un resumen de las interacciones encontradas como factores explicativos del ecosistema emprendedor del sector hotelero se tiene que la orientación, intención y comportamiento propios del emprendedor condicionan la legitimidad del emprendimiento, especialmente la intención emprendedora. Por una parte, los factores institucionales parecen condicionar fundamentalmente el comportamiento emprendedor; por la otra, la legitimidad tributa a la reputación.

Segmentación de emprendedores en el ecosistema emprendedor del sector hotelero de Guayaquil. La proyección de los respondientes en el plano factorial resultante de los dos primeros ejes conforma cinco agrupaciones de emprendedores, y al valorar la disposición de cada uno de los grupos se puede comentar, que los grupos I, II, III y $\checkmark$ se disponen sobre el gradiente que recorre el primer eje de izquierda a derecha (figura 15). Eso se interpreta por orientarse a desafiar la competencia desde la intención de conformar equipo de trabajo que conlleven a un aprovechamiento de las oportunidades de mercado y la tecnología que los legitímese al realizar actividades pertinentes y eficaces en aras de las metas de la organización.

Así al referir el primer eje, el grupo I percibe esos elementos en los más altos puntajes; en tanto el grupo II y el grupo IV ya perciben pequeñas brechas en comparación con la excelencia (figura 17). El grupo III como tendencia valora dos puntos por debajo de la excelencia las variables explicativas del primer eje y, por último, el grupo $\mathrm{V}$ reporta los peores valores en casos hasta el extremo inferior (figura 15).

Al referir al segundo eje, los grupos I y III reportan percepciones altas sobre las variables que definen ese eje, mientras que los grupos II y $V$ muestran una diversidad de percepciones nunca más allá de tres unidades por debajo de la excelencia, el grupo IV tiene las peores percepciones de las variables que lo determinan.

Entonces, los factores de apoyo institucional y saber gestionar el riesgo y la información para emprender, inciden en la autonomía de los empleados en la 
Revista Ciencia \& Tecnología No. 16, 31 de octubre de 2017 ISSN impreso: 1390 - 6321

\section{Jornada de Investigación Universidad Tecnológica Empresarial de Guayaquil}

búsqueda de oportunidades para poder visualizar y aprovechar las nuevas oportunidades, y son valorados por la mayoría de los emprendedores entre el máximo y dos o tres valores por debajo de ése. Solo el grupo IV tiene los valores más bajos.

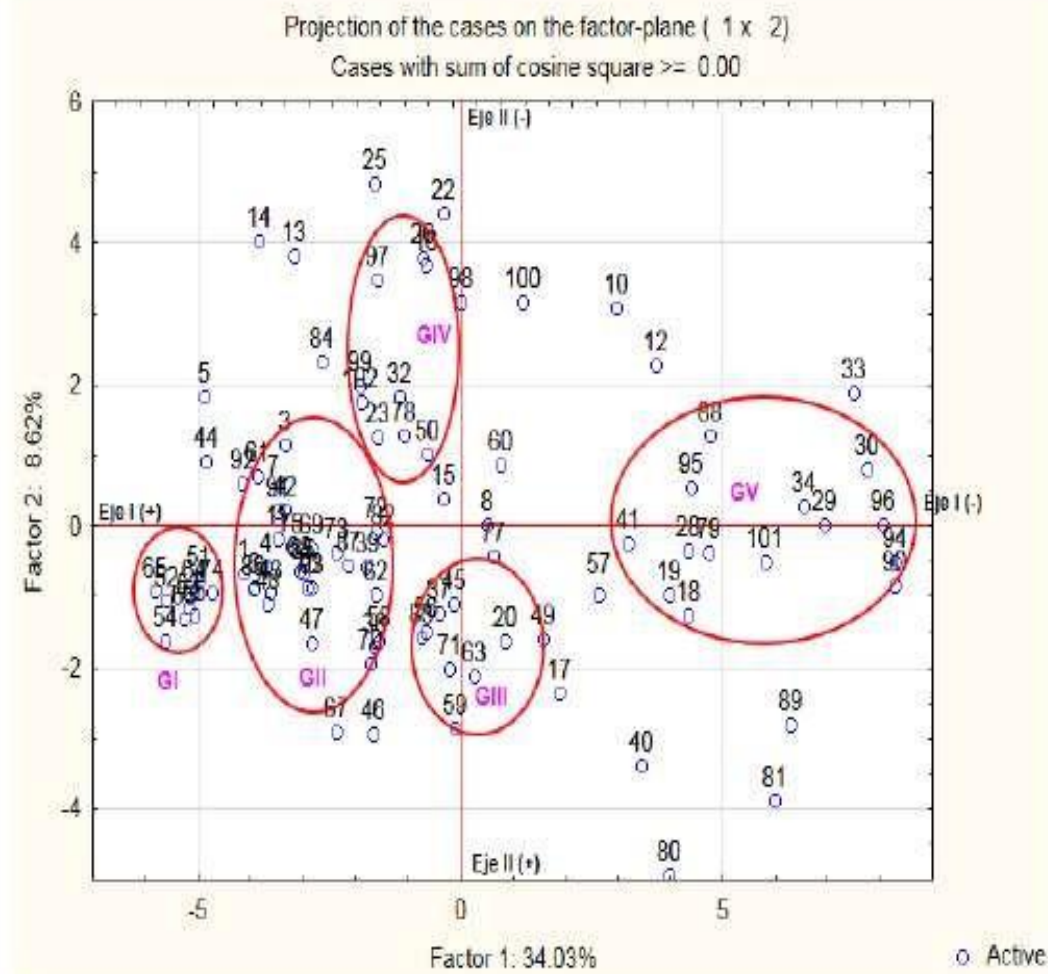

\section{Gráfico 15: Proyección de los respondientes en el plano factorial compuesto por el primer y segundo eje}

Fuente: Salida del STATISTICA

Todo lo anterior hace que los grupos I y III sean grupos concentrados, por los efectos del segundo eje los grupos II y IV se alargan hacia arriba; en tanto, el primer factor provoca la dispersión en el grupo $\mathrm{V}$ al estilarlo en el sentido de izquierda a derecha.

Esa combinación deriva cuatro tipologías de emprendedores (figura 15):

GI: Los factores que condicionan el ecosistema emprendedor son favorables al emprendimiento, donde de reportan las mayores puntuaciones.

GII: Se orienta al desafío de la competencia con la intención de conformar equipo de trabajo, aprovecha las oportunidades de forma que den legitimidad en actividades que tributen a las metas de la organización. En los factores institucionales resalta el saber gestionar el riesgo y la información que deriven en una orientación de autonomía a los empleados para que aporten oportunidades que hagan que el emprendedor visualice y aproveche las nuevas oportunidades, poseen pequeñas brechas en relación a lo ideal. 


\section{Jornada de Investigación Universidad Tecnológica Empresarial de Guayaquil}

GIII: Se orienta al desafío de la competencia con la intención de conformar equipo de trabajo, aprovecha las oportunidades de forma que den legitimidad en actividades que tributen a las metas de la organización se percibe con una brecha de algo más de un punto con respecto al ideal. Mientras que en los factores institucionales se destacan saber gestionar el riesgo y la información que deriven en una orientación de autonomía a los empleados para que aporten oportunidades que hagan que el emprendedor visualice y aproveche las nuevas oportunidades se perciben con una brecha menor con el ideal.

GIV: Tienen una baja percepción de los factores institucionales de saber gestionar el riesgo y la información que deriven en una orientación de autonomía a los empleados, para que aporten oportunidades que hagan que el emprendedor visualice y aproveche las nuevas oportunidades, pero una percepción de relativas pequeñas brechas en la orientación al desafío de la competencia con la intención de conformar equipo de trabajo, aprovecha las oportunidades de forma que den legitimidad en actividades que tributen a las metas de la organización tiene alrededor de dos puntos menos de lo ideal.

GV: Poseen una baja percepción de los factores institucionales de saber gestionar el riesgo y la información que deriven en una orientación de autonomía a los empleados para que aporten oportunidades que hagan que el emprendedor visualice y aproveche las nuevas oportunidades entre alta y alrededor de dos categorías por debajo, pero una percepción de relativamente alta de las brechas en la orientación al desafío de la competencia con la intención de conformar equipo de trabajo, aprovecha las oportunidades de forma que den legitimidad en actividades que tributen a las metas de la organización tiene alrededor de un punto menos de lo ideal.

En cuanto al perfil de emprendedores de esos grupos, se puede anotar que los grupos I y II son más típicos de hoteles posicionados en el mercado o con respaldo de alguna cadena hotelera, mientras que en el grupo $\mathrm{V}$ se ubican los emprendimientos con ofertas o productos menos desarrollados. Mientras que el grupo IV incluye aquellos emprendimientos que se encuentran en fase de desarrollo los que deben de someterse a la competencia con los más desarrollados y cierto reconocimiento en el mercado.

\section{Conclusiones}

Una vez expuesto y discutido los resultados encontrados en esta investigación se muestran a las siguientes conclusiones. Al analizar los aportes teóricos de diferentes autores sobre los factores que condicionan el ecosistema emprendedor se evidenció que la Teoría Institucional es propicia y pertinente para abordar este tema.

La metódica concebida en esta investigación puede ser patrón para investigaciones similares, al evidenciar su factibilidad, validez y consistencia de la misma.

Al determinar los factores que condicionan el ecosistema emprendedor del sector hotelero de Guayaquil se obtuvo que en ocho ejes logran explicar el $68.3 \%$ de la varianza total. Esos son: Orientación, intención y comportamiento del emprendedor que condicionan la legitimidad de la organización.

Factores institucionales dados en el entorno del ecosistema emprendedor, que 


\section{Jornada de Investigación Universidad Tecnológica Empresarial de Guayaquil}

condicionan la orientación en la autonomía y la visualización y aprovechamiento de las oportunidades como parte de la creatividad del emprendedor.

La intención de identificar una oportunidad y establecer una empresa, pasa por elementos subjetivos del emprendedor y la aprobación social de esa toma de decisiones: contraposición del factor institucional normativo de la admiración social de los emprendedores con el comportamiento de asistir a acciones de formación; contraposición de la legitimidad de las actividades en aras de las metas de la organización con los elementos de intención emprendedora; contraposición de la autoeficacia de detectar nuevos productos y áreas potenciales de mercado con la intención de elaborar plan de negocio y conformar equipo de trabajo; contraposición de la legitimidad de que las actividades que se desarrollen contribuyan o no a la meta de la organización con la reputación como parte de la cultura del país; y, reputación como acción que es apoyada por la sociedad.

Al caracterizar la muestra de emprendedores encuestados- como fuente primaria de la investigación- se constató su representatividad de la diversidad de características de emprendedores y emprendimientos en el ecosistema emprendedor del sector hotelero de Guayaquil. Así como que los factores del ecosistema emprendedor reportan índices ponderados de percepción que se pueden calificar de buenos, con valores más bajos en los factores institucionales.

Al segmentar los emprendedores en el ecosistema emprendedor del sector hotelero de Guayaquil a partir de su proyección en el plano factorial conformado por el primer y segundo eje se conforman cinco grupos de respondientes. Los grupos formados fueron:

Grupo I: Emprendedores que valoran como favorable (totalmente de acuerdo) casi todos los factores que condicionan el ecosistema emprendedor.

Grupo II: Emprendedores que valoran los factores que condicionan el ecosistema emprendedor en puntuaciones cercanas al máximo (totalmente de acuerdo a muy de acuerdo).

Grupo III: Emprendedores que valoran los factores que condicionan el ecosistema emprendedor en puntuaciones cercanas al máximo (muy de acuerdo a de acuerdo).

Grupo IV: Emprendedores con baja percepción de los factores institucionales (de acuerdo a en desacuerdo) y de la orientación emprendedora (de acuerdo a ni de acuerdo ni en desacuerdo). Pero relativamente alto en los demás aspectos (muy de acuerdo).

Grupo V: Emprendedores con baja percepción de prácticamente todos los factores (de acuerdo a ni de acuerdo ni en desacuerdo).

\section{Referencias bibliográficas}

Ashford, B. \& Gibbs, B. (1990). The double-edge of organizational legitimation. Organization Science, vol.1, n², pp.117-194. 


\section{Jornada de Investigación Universidad Tecnológica Empresarial de Guayaquil}

Brush, C. G., \& Chaganti, R. (1998). Businesses without glamour? An analysis of resources on performance by size and age in small service and retail firms. Journal of Business Venturing 14, 233-57.

DiMaggio, P.J. \& Powell, W.W. (1983). The Iron Cage Revisited: Institutional Isomorphism and Collective Rationality. American Sociological Review, vol.48, pp.147-160.

Fishbein, M. \& Ajzen, I. (1975). Belief, attitude, intention, and behavior. An introduction to theory and research. Addison-Wesley, New York.

Hidalgo, G., Kamiya, M. \& Reyes, M. (2014). Emprendimientos dinámicos en América Latina. Avances en prácticas y políticas. Banco de Desarrollo de América Latina. Serie Políticas Públicas y Transformación Productiva, $N^{\circ} 16$.

Liu, A. \& Wall, G. (2006). Planificación del turismo empleo: perspectiva de un país en desarrollo. Gestión turística, 27, 159-170.

Low, M.B. and McMillan, I.C. (1988). Entrepreneurship: Past research and future challenges. Journal of Management, Vol. 14, No. 2, pp. 139-161

Mestre Soler, J. R. (1999): Técnicas de Gestión y Dirección Hotelera. Barcelona: Ediciones Gestión 2000.

Meyer, J.W. \& Rowan, B. 1977. Institutionalized Organizations: Formal Structure as Myth and Ceremony. American Journal of Sociology, vol.83, pp.340-363.

North, D. 1990. Institutions, institutional change and economic performance. Political economy of institutions and decisions. Cambridge University press.

ProChile (2014). Estudio de mercado del Ecosistema de Innovación en Los Ángeles, Estados Unidos. Ministerio de Relaciones Exteriores de Chile.

Rao, H. (1994). The social construction of reputation: Certification contests, Legitimation, and the Survival of organizations in the American Automobile Industry. Strategic Management Journal, Vol. 15. Special Issue: Competitive Organizational Behavior, 29-44

Rutherford, M. (1995). The Old and the New Institutionalism: Can bridges be built? Journal of Economic Issues, vol. XXIX, no2, pp.443-451.

Schuman, M.C. (1995). Managing Legitimacy: Strategic and Institutional approaches. Academy of Management Review, vol.20, n03, pp.571-610.

Schumpeter, J.A. (1934). The theory of economic development. Cambridge: Harvard University Press.

Scott, W.R. (1995). Institutions and Organizations. Ideas, Interests and Identities. 


\section{Jornada de Investigación Universidad Tecnológica Empresarial de Guayaquil}

Sage Publications. Paperback: 360 pages Publisher: Sage (1995) Management, 17 (2), 136-140.

Stevenson, H. \& Gumpert, D. (1985). The heart of Entrepreneurship. Harvard Business Review, Vol. 63, No.2, pp. 85-94.

Venkataraman, S. (1997): The distinctive domain of entrepreneurship research: An editor's perspective, in J. Katz y R. Brockhaus (editores), Advances in Entrepreneurship, Firm Emergence, and Growth, 3, Greenwich, CT, US: JAI Press, 